Article

\title{
Effect of Vibration to Fatigued Plantar Flexor Muscles on Pos- tural Stability in Healthy Young Adults
}

\author{
Parth Doshi ${ }^{1}$, Isha Akulwar-Tajane ${ }^{1,{ }^{*}}$ \\ ${ }^{1}$ K. J. Somaiya College of Physiotherapy, Maharashtra, India \\ * Correspondence: drishasa@yahoo.co.in; Tel.: +919769490996
}

How to cite this paper: Doshi, P., \& Akulwar-Tajane, I. (2021). Effect of Vibration to Fatigued Plantar Flexor Muscles on Postural Stability in Healthy Young Adults. Universal Journal of Sport Sciences, 1(1), 11-18. DOI: 10.31586/ujss.2021.010102. Retrieved from https://www.scipublications.com/journal/in-

dex.php/ujss/article/view/8

Received: May 15, 2021

Accepted: June 21, 2021

Published: June 22, 2021

Copyright: (c) 2021 by the authors. Submitted for possible open access publication under the terms and conditions of the Creative Commons Attribution (CC BY) license (http://creativecommons.org/licenses /by/4.0/).

\begin{abstract}
Objective: The effects of muscle fatigue appear to elicit a transient means of postural instability; however, the recovery of postural stability after fatiguing exercise has not been extensively studied. This study aimed to determine the immediate effect of local vibration applied to fatigued plantar flexor muscles on postural stability in healthy young adults. Design: True Experimental (pre-test - post test design with a control group). Setting: A tertiary care centre. Participations: 42 healthy young adults. Intervention: Fatigue of plantar flexors muscles was induced by repeated dynamic contractions performed to maximum exhaustion. Experimental group $(n=21)$ received local vibration to fatigued muscles with a handheld vibrator whereas the control group $(n=21)$ received no intervention (rest). Postural stability was assessed using one leg stance test (OLST) under three different conditions viz. pre-fatigue, post-fatigue and post rest/vibration. Results: Comparative analysis of postural stability within the group was done pre-fatigue, post-fatigue and post rest/vibration condition using Kruskal-Wallis test. A significant decrease from pre-fatigue to postfatigue condition ( $\mathrm{p}$-value $<0.001$ ); and a significant increase from post-fatigue to post rest/vibration condition ( $p$-value $<0.001$ ) was observed in OLST in both - control group and experimental group. However, further comparison of OLST between the groups (using unpaired t test) showed a significant difference in post-fatigue condition versus post rest/vibration condition in favour of the experimental group. Conclusion: Plantar flexors fatigue significantly affects one leg stance time in healthy young adults. Results of this study show that there is an immediate effect of vibration to fatigued muscles on recovery of postural stability in healthy young adults. Recovery of postural stability achieved quickly with such a simple tool may help increase the degree of participation in sports and other similar activities which can cause muscles to fatigue. However, more studies are required to determine this effect using other clinical measures.
\end{abstract}

Keywords: Muscle fatigue, Postural stability, Local vibration, Plantar flexors, One leg stance time

\section{Introduction}

Postural control is a complex task that requires the integration of visual, vestibular and somatosensory inputs from all over the body to assess the position and motion of the body in space and the ability to generate forces to control body position [1]. The task of maintaining upright balance is a critical component in performing most daily activities. To perform daily activities and to keep up the performance muscles need to work at their optimum level. As the muscle is working continuously to keep up with the demands, with time the muscle fatigues and the performance is decreased; thus, affecting the postural control and balance of the human body.

Muscle fatigue can be defined as "an exercise-induced reduction in the ability of muscle to produce force or power whether or not the task can be sustained [2]." Numerous studies have investigated the effects of muscular fatigue on the regulation of bipedal quiet upright standing. Generally, a deterioration of postural control with muscular fatigue was reported. Considering postural control as a sensorimotor process, it is conceivable that 
muscular fatigue, known to alter the peripheral proprioceptive system, the central processing of proprioception but also the force-generating capacity, affect both the sensory and the motor side of the process [3]. Especially in sports players, fatigue may lead to detrimental performance and loss of balance; and make the body prone to falls and other musculoskeletal injuries. This in turn may have a severe impact on their quality of life and social costs.

The effects of muscle fatigue appear to elicit a transient means of postural instability however the recovery of postural stability after fatiguing exercise has not been extensively studied. There are different recovery methods to treat fatigue but vibration is one of the lesser explored and lesser-known fields. Since the vibration can be applied with a wide spectrum of frequencies and settings, different effects on healthy and pathologic tissues are possible. However only a few studies described specific vibrational training protocols, and this lack of information generates uncertainties regarding the effectiveness of vibration on muscle fatigue and balance [4]. This reflects the wide controversy regarding expected outcomes. Understanding the physiology of vibration on the human body is very much important to find out the effect of vibration on the fatigued muscles.

Vibration therapy works on the pain gate mechanism to block the nociceptive receptors by blocking the transmission of pain stimulus. Therefore, applied mechanical vibration can have a summative effect, with other pain control strategies, in reducing behavioural and physiological pain responses [5]. In vibration therapy, the stimulation of muscle spindles and alpha-motor neurons causes muscle contraction and it increases electro-myographic activity. Oxygen consumption, muscle temperature and skin blood flow increase directly proportional to vibration [6]. These already established physiological mechanisms need clinical and scientific evidence to show that vibrational therapy will be useful and will be the future of the training or treatment protocol. Based on this information we hypothesized that there could be a significant difference in one leg stance time after local vibration to fatigued plantar flexor muscles in healthy young adults.

\section{Methodology}

Approval for the research study was obtained from the Institutional Ethics Committee of K J Somaiya College of Physiotherapy and the study procedures accord with the ethical guidelines. It was a true experimental study with pre-post design. A volunteer sample of university students was recruited using a convenient sampling method after screening for the eligibility criteria. As per the calculated sample size of 42 , eligible subjects were randomly selected using a computer-generated table; and randomly allocated to two groups viz. Experimental group and Control group each having 21 subjects. The study participants were healthy young females in the age group of 18-25 years and BMI of $18.5-22.9 \mathrm{Kg} / \mathrm{m}^{2}$. Informed written and verbal consent was obtained prior to their enrolment. Individuals suffering from any musculoskeletal or neurological condition/s which can affect balance; having congenital anomalies or acquired deformities of the lower extremity, open wounds near plantar flexor muscles, uncorrected visual impairments, vestibular impairments, or detected with any metabolic or endocrine conditions that can influence fatigue were excluded from participation.

All the 42 participants were assessed under 3 conditions namely pre-fatigue, immediately post-fatigue and post-rest/vibration. Before the assessment stability leg was identified by a ball kick test. The leg by which the subject kicked the ball was considered as the mobility leg and the other leg was considered as the stability leg. Assessment of OLST and intervention of vibration was applied to the stability leg.

In pre-fatigue condition, single leg stance time of stability leg was assessed. Fatigue of plantar flexor muscles of the stability leg was induced by repetitive unilateral heel raises in the standing position, performed to maximum. Subject was instructed to perform the movement as many times as possible till she was unable to continue due to muscle fatigue. Verbal encouragement was given to ensure that the subject worked maximally. A digital 
metronome set at 40 beeps/min was used to ensure that all the subjects performed the heel raises at a consistent rate. Once the fatigue of muscle was set in it was graded on the Borg scale [7] between 6-20 and the One Leg Stance Time (OLST) [8] was assessed immediately.

In the post-fatigue condition, both the group participants were made to lie in supine with lower extremity well supported and in a non-weight bearing position. For the experimental group participants, vibration was applied to plantar flexors (at musculo - tendinous junction) of the stability leg for 5 minutes with a hand-held vibrator at $50-60 \mathrm{~Hz}$ frequency. The control group participants while taking rest were instructed to avoid any muscular activity of the lower extremity muscles. OLST of the stability leg was immediately assessed immediately after 5 minutes of rest/vibration for the control/experimental group respectively.

\section{Outcome measure}

\subsection{One leg stance time (OLST)}

For OLST, the subject stood barefoot on a firm stable surface on the dominant stability leg with the other leg raised, arms crossed over the chest. Test was performed under eyes closed condition to avoid vision from interfering with the task of postural control. Time of one leg standing was recorded in seconds using a stopwatch. Test was terminated when the subject used her arms (i.e., uncrossed arms), used the raised foot (moved it toward or away from the standing limb or touched the floor) moved the weight-bearing foot to maintain balance (i.e., rotated foot on the ground), or opened eyes. Three trials were given and the best of the three trials was taken as the final reading.

OLST was assessed under three conditions:

- Condition 1: Pre-fatigue

- Condition 2: Post-fatigue (immediately after fatigue has set in)

- Condition 3: Post-recovery - Control group - after 5 minutes of rest - Experimental group -after 5 minutes of vibration

All the assessments were carried out under standardized conditions. Subjects were asked to refrain from any strenuous physical activity for the past 48 hours. Also, use of any medication which can have an effect on neuromuscular fatigue or postural control was prohibited for the past 24 hours. Considering the time-of- day effects on fatigue and postural control, assessment was carried out during the same time of the day (afternoon) for all the subjects. All the trials were performed during a single experimental session. All the tests were administered by the same investigator and in an identical manner under all three conditions. Data thus collected was subjected to statistical analysis.

\section{Results}

This sample consisted of females with a mean age of $19.8( \pm 2.13 \mathrm{SD})$ and BMI $21.10 \pm$ 1.28. The dominant leg for stability was left sided for $92.86 \%$ and right sided for the remaining $7.14 \%$ as determined using ball kick test. None of the participants exhibited mixed dominance.

In this experimental design, rest/vibration served as an independent variable and OLST as the dependent variable. Comparative analysis was done under three different conditions viz. pre-fatigue, post-fatigue and post-recovery (rest/vibration) for the control and experimental group separately. Data did not pass normality test so nonparametric test i.e., Kruskal-Wallis test was used for the repeated measures within-group analysis. Data for difference of mean showed normal distribution and thus was analysed using a parametric test i.e., unpaired $t$ test. Statistical significance was set at $p<0.001$ (one-tailed) for all the analyses.

Within group comparison of OLST showed a significant decrease from pre-fatigue to post-fatigue condition; and a significant increase from post-fatigue to post rest/vibration condition in both - control group and experimental group respectively (Table 1). However, 
a further comparison between the groups showed a significant difference of mean of OLST in post-fatigue condition versus post vibration/rest condition in favour of experimental group (Table 2). These results reject the null hypothesis in view of significant improvement in the experimental group moreover the control group.

Table 1. Comparative analysis between three experimental conditions in both the groups

\begin{tabular}{|c|c|c|c|c|c|}
\hline $\begin{array}{l}\text { Outcome } \\
\text { measure }\end{array}$ & \multicolumn{3}{|c|}{ Condition } & \multicolumn{2}{|c|}{$\begin{array}{l}\text { Kruskal-Wallis test } \\
\quad(P \text { Value }<0.05)\end{array}$} \\
\hline OLST & $\begin{array}{l}\text { Pre-fatigue } \\
(\text { mean } \pm S D)\end{array}$ & $\begin{array}{l}\text { Post-fatigue } \\
(\text { mean } \pm \text { SD) }\end{array}$ & $\begin{array}{l}\text { Post-Vibra- } \\
\text { tion/Rest(mea } \\
\quad \mathrm{n} \pm \mathrm{SD})\end{array}$ & $\begin{array}{l}\text { Comparison be- } \\
\text { tween Pre- \& Post- } \\
\text { fatigue }\end{array}$ & $\begin{array}{c}\text { Comparison between } \\
\text { Post-fatigue \& Post-Vi- } \\
\text { bration/Rest }\end{array}$ \\
\hline $\begin{array}{l}\text { Experimental } \\
\text { group }\end{array}$ & $59.47 \pm 32.02$ & $28.28 \pm 16.91$ & $83.09 \pm 40.87$ & $\begin{array}{c}\mathrm{p} \text { value }= \\
\text { Significant decrease }\end{array}$ & $\begin{array}{c}\mathrm{p} \text { value }= \\
\text { Significant increase }\end{array}$ \\
\hline Control group & $59.95 \pm 35.86$ & $27.33 \pm 18.77$ & $50.14 \pm 23.75$ & Significant decrease & Significant increase \\
\hline
\end{tabular}

Table 2. Comparison between mean of OLST of both the groups in post-fatigue versus post -vibration/rest

\begin{tabular}{|c|c|c|c|}
\hline & $\begin{array}{c}\text { Mean } \pm \text { SD of difference of } \\
\text { OLST (Post-vibration /rest) } \\
\text { and (Post-fatigue) }\end{array}$ & Statistical test used & $\begin{array}{c}\text { Comparison between mean of both } \\
\text { the groups in Post-fatigue versus } \\
\text { Post-vibration/rest }\end{array}$ \\
\hline $\begin{array}{c}\text { Experimental } \\
\text { Group }\end{array}$ & $54.81 \pm 31.27$ & \multirow{2}{*}{ Unpaired T test } & \multirow{2}{*}{$\begin{array}{l}\mathrm{p} \text { value }=0.0006 \\
\text { A significant difference in favour of } \\
\text { experimental group }\end{array}$} \\
\hline $\begin{array}{l}\text { Control } \\
\text { Group }\end{array}$ & $22.95 \pm 21.93$ & & \\
\hline
\end{tabular}

\section{Discussion}

In this experimental study, fatigue was induced in the plantar flexor muscles and subsequently its recovery with local vibration was analysed in healthy young adults aged 18-25 years. Results of the study show that vibration applied to the musculotendinous junction of fatigued ankle plantar flexor muscles for 5 minutes immediately increases the OLST when compared to a control group.

Fatigue is defined as an inability to maintain a power output or force during repeated muscle contractions [10]. It is the inability to maintain muscle tension when a contraction is sustained or reproduce a particular tension during contractions over time [11]. Fatigue of the plantar flexors showed a detrimental effect on postural stability as evident from a significant decrease in OLST from a pre-fatigue to post-fatigue condition. Postural control is a person's ability to maintain an appropriate relationship between the body segments; and between the body and the environment; and to keep the body's centre of mass over the base of support when performing a task [12]. Balance is the ability to keep the body in 
equilibrium in either static or dynamic positions with minimal muscle activity [13]. Williams (1983), described balance as a state in which the opposing internal and external forces are in a state of equilibrium [14] Balance control is multidimensional and requires complex sensory, neuromuscular, and central processing systems [15] The ability to maintain balance depends on visual, vestibular, auditory, stomato-sensory and motor systems. [16] The observed decrease in the OLST can be due to altered proprioception in muscle receptors and even reduction in ATP production as aerobic capacity of the muscle is taken over by anaerobic pathway, which accumulates lactic acid in the muscle and thus depleting the muscle for a sustained contraction to maintain OLST for long time. According to Simoneau et al. (2006) moderate fatigue led to an immediate decrement of the performance on the balance control task [17] Nordone et al. (1997) reported similar findings where body sway was increased after strenuous physical exercise [18] This was also confirmed by Eva et al. (1999), who reported that short term sub-maximal cycling had an effect on balance in single limb stance tests [19] This study shows that there is a significant effect of fatigue on OLST i.e. static postural stability.

After 5 minutes of rest/vibration post-fatigue, there was a significant improvement in OLST in both the groups but the experimental group showed a better result when compared to the control group. Boucher et al showed that there is significant improvement in neuromuscular control of a muscle after local vibration to back extensor muscle. [20] Hagbarth et al (1996) showed that local vibration on a muscle stimulates the muscle spindle receptors and induces a tonic vibration reflex. It even causes alpha motor neurons to get activated causing muscle to contract and increase the electromyographic activity of the muscle [9] and recruit more muscle fibres for stronger contraction. This causes better motor unit synchronisation and improvement of synergistic muscle coordination which leads to improvement in muscle explosive power. The study shows that increase of contractile ability in muscular areas subject to vibration exercise induces an evident right shift of force velocity curve and of force power one.[9,21] showed that local repeated Muscle Vibration $(\mathrm{rMV})$ when applied to muscle shows improvement in leg power by $35 \%$, improved stance performance and effect lasted for 90 days after the rMV treatment.[21] Vibration is a simple, easy and a non-invasive tool used for the treatment and can give us quick results as shown by the study. However, there are articles saying the effect of vibration is short lasting. [22-24]

We also believe that the effect might not last long as there are various factors in vibration protocol on which the duration of the effect depends. A plastic change in nervous networks can be supposed since proprioceptive circuits are a plastic substratum on which it is possible to act with different forms of conditioning paradigms, based on proprioceptive stimulation. [23,24,25,26,27] In fact, short or long-term (i.e. minutes or months) plastic changes of nervous circuits [28] can be induced by simple or complex combinations of sensory inputs.[28] Applied vibration frequency $(100 \mathrm{~Hz})$ is reported to be adequate to induce persisting effects in nervous circuits.[23,24] Exposure time to stimulation is also an important factor [27] and, in our experimental set up, the 5 minutes exposure for one time might constitute an inadequate condition to develop a long-term enhancement in nervous circuitry. Moreover, different research studies suggest that it is possible to produce plastic changes in central nervous circuitry by combining the subject's attention with different forms of vibratory stimulation $[25,26]$ and that such protocols can improve the sensorial discrimination [26]. In this research, there was an improvement in stance performance when compared to the control group but the duration of the vibration effects was not studied. Muscle vibration is considered a powerful stimulus for Golgi and spindle afferents.[29] Moreover, the applied frequency is considered optimal for primary spindle afferents.[30] However, the very low values of the applied vibration displacement introduce the question on whether primary muscle spindle afferents can be excited.

There is a lack of studies in healthy young adults and even on the effect of vibration on fatigue, thus, more studies are required to understand the effect of vibration on the 
human body. Though there are articles showing long term effects and improvement in performance, all those studies include various vibration protocols for longer duration of treatment and show the plastic changes in cortex also affecting the proprioceptive system. These observations suggest that focal vibration can also reduce the risk of falls [31] but this should be investigated through a stability study under dynamic conditions, such as sway perturbations or walking, and a longitudinal study on the risk of falls. To obtain comparable increases in motor performance, many protocols are based on physical exercises and are defined by combining trials for muscle strengthening and balance $[32,33,34,35,36]$ Furthermore, vibratory stimulation does not impose loads on muscles and joints, while intense physical exercises may cause injuries in elderly patients.[37] Finally, this treatment is structured as one 5-minute session. Recovery of postural stability achieved quickly with such a simple tool and with ease of application of hand-held vibrator suggest its use in sports field as alternative/complementary training method to improve muscle performance by activating muscle spindles. This may help to increase degree of participation in sports and other similar activities which can cause muscle to fatigue or may even hamper postural stability in these activities and indirectly affects the performance. Given the benefits of this experiment, combined with the safety of this procedure and the minimal time required to complete it, local muscle vibration may represent an ideal low-cost treatment for people involved in physical activities which may induce fatigue or elderly in whom fatigue can be a factor predisposing them to falls.

However, we acknowledge several limitations, the most important being the subjective nature of the criteria used for muscle fatigue. Also, fatigue induced experimentally may differ from the fatigue experienced in functional/sports activities. Another limitation is that muscle activity and/or its force generation capacity post intervention was not assessed in this study. We recommend future studies focusing on more objective tools and methods and incorporating additional measures of muscle dynamics. The effect of vibration on fatigued muscles and dynamic postural stability can be studied using other clinical measures. Moreover, the time period for which the effect of vibration persists needs to be studied.

Nonetheless, to our knowledge, the current study is the first one to investigate the effect of vibration in recovery of postural stability from a fatiguing condition. Considering the limited literature available in this regard, more studies are warranted to explore these results further.

\section{Conclusion}

Plantar flexor muscle fatigue significantly affects one leg stance time in healthy young adults. There is an immediate positive effect of vibration to fatigued muscles on recovery of postural stability in healthy young adults. Vibration is a simple, easy and a non-invasive tool and can have potential application for muscle recovery in sports.

\section{Abbreviations}

1. OLST- One Leg Stance Time.

2. rMV - repeated Mechanical Vibration.

\section{Declarations}

1. Ethics approval and consent to participate- An ethical approval was granted by the Institutional Ethics Committee of K J Somaiya College of Physiotherapy. The procedures followed protocols and accord with the ethical standards of the institutional review board. A written informed consent was taken from all the participants at the beginning of the study.

2. Consent for Publication- Not Applicable. 
3. Availability of Data and Materials- All data generated or analysed during this study are included in this article [and its supplementary information files].

4. Competing interests-The authors declare that there are no competing interests.

5. Funding-The authors have not received any kind of financial support in the conduct or publication of this research.

6. Authors' contributions-Both the authors Parth Doshi and Isha Akulwar-Tajane have nearly equal contributions to the conception and design of the work; the acquisition, analysis or interpretation of data for the work; drafting the work, revising it critically for important intellectual content; and final approval of the version to be published. Specific contribution of Parth is in the acquisition of the data and Isha contributed to the data analysis and interpretation. All authors have read and approved the manuscript.

7. Acknowledgments-The authors acknowledge the valuable contribution of the study participants; and support of the principal and research committee of the institute in the conduct of the study.

\section{References}

[1] Akulwar I, Mulgaonkar S. Effect of Trunk Extensor Muscle Fatigue on Postural Stability in Healthy Young Adults. 2015; 6(7):5.

[2] Wan J, Qin Z, Wang P, Sun Y, Liu X. Muscle fatigue: general understanding and treatment. Exp Mol Med. 2017 Oct; 49(10):e384e384.

[3] The magnitude of the effect of calf muscles fatigue on postural control during bipedal quiet standing with vision depends on the eye-visual target distance. Gait Posture. 2006 Oct 1; 24(2):169-72.

[4] Cerciello S, Rossi S, Visona E, Corona K, Oliva F. Clinical applications of vibration therapy in orthopaedic practice. Muscles Ligaments Tendons J. 2016 May 19; 6(1):147-56.

[5] Caylor J, Reddy R, Yin S, Cui C, Huang M, Huang C, et al. Spinal cord stimulation in chronic pain: evidence and theory for mechanisms of action. Bioelectron Med. 2019 Jun 28; 5(1):12.

[6] Faust KA. The acute effect of whole-body vibration on gait parameters in adults with cerebral palsy. Title on signature form: Acute effects of whole-body vibration on gait parameters in adults with cerebral palsy [Internet]. 2011 Dec 17 [cited 2021 Jan 23]; Available from: http://cardinalscholar.bsu.edu/handle/123456789/195163

[7] Parameterization and reliability of single-leg balance test assessed with inertial sensors in stroke survivors: a cross-sectional study [Internet]. [Cited 2021 Jan 23]. Available from: https://www.ncbi.nlm.nih.gov/pmc/articles/PMC4158088/

[8] Pfeiffer KA, Pivarnik JM, Womack CJ, Reeves MJ, Malina RM. Reliability and validity of the Borg and OMNI rating of perceived exertion scales in adolescent girls. Med Sci Sports Exerc. 2002 Dec; 34(12):2057-61.

[9] Burke D, Hagbarth KE, Löfstedt L, Wallin BG. The responses of human muscle spindle endings to vibration during isometric contraction. J Physiol. 1976; 261(3):695-711.

[10] Muscular Exercise and Fatigue | SpringerLink [Internet]. [Cited 2021 Jan 24]. Available from: https://link.springer.com/article/10.2165/00007256-198502020-00004

[11] Fox SI. Laboratory guide to human physiology: concepts and clinical applications. W.C. Brown; 1976. 346 p.

[12] Shumway-Cook A, Woollacott MH. Motor Control: Theory and Practical Applications. Williams \& Wilkins; 1995.496 p.

[13] Thomson AM, Skinner A, Piercy J. Tidy's Physiotherapy. Butterworth-Heinemann; 1991. 501 p.

[14] Williams HG. Perceptual and Motor Development. Prentice-Hall; 1983. 360 p.

[15] Fabunmi AA, Lesi FEA, Akosile CO. Effect of Exercise Induced Fatigue on Standing Balance Performance. Sport Exerc Health Fit Wellness. 2011; 61.

[16] Iverson BD, Gossman MR, Shaddeau SA, Turner ME Jr. Balance Performance, Force Production, and Activity Levels in Noninstitutionalized Men 60 to 90 Years of Age. Phys Ther. 1990 Jun 1; 70(6):348-55.

[17] Simoneau M, Bégin F, Teasdale N. The effects of moderate fatigue on dynamic balance control and attentional demands. J NeuroEngineering Rehabil. 2006 Sep 28; 3:22.

[18] Nardone A, Tarantola J, Giordano A, Schieppati M. Fatigue effects on body balance. Electroencephalogr Clin Neurophysiol Mot Control. 1997 Aug 1; 105(4):309-20.

[19] Ageberg E, Roberts D, Holmström E, Fridén T. Balance in single-limb stance in healthy subjects - reliability of testing procedure and the effect of short-duration sub-maximal cycling. BMC Musculoskelet Disord. 2003 Jun 27; 4(1):14.

[20] Boucher J-A, Abboud J, Nougarou F, Normand MC, Descarreaux M. The Effects of Vibration and Muscle Fatigue on Trunk Sensorimotor Control in Low Back Pain Patients. PLOS ONE. 2015 Aug 26; 10(8):e0135838.

[21] Filippi GM, Brunetti O, Botti FM, Panichi R, Roscini M, Camerota F, et al. Improvement of Stance Control and Muscle Performance Induced by Focal Muscle Vibration in Young-Elderly Women: A Randomized Controlled Trial. Arch Phys Med Rehabil. 2009 Dec; 90(12):2019-25. 
[22] Kawakami Y, Miyata M, Oshima T. Mechanical vibratory stimulation of feline forepaw skin induces long-lasting potentiation in the secondary somatosensory cortex. Eur J Neurosci. 2001 Jan; 13(1):171-8.

[23] Differential effect of muscle vibration on intracortical inhibitory circuits in humans [Internet]. [Cited 2021 Jan 24]. Available from: https://www.ncbi.nlm.nih.gov/pmc/articles/PMC2343209/

[24] Rosenkranz K, Rothwell JC. The effect of sensory input and attention on the sensorimotor organization of the hand area of the human motor cortex. J Physiol. 2004 Nov 15; 561(Pt 1):307-20.

[25] Recanzone GH, Merzenich MM, Jenkins WM, Grajski KA, Dinse HR. Topographic reorganization of the hand representation in cortical area 3b owl monkeys trained in a frequency-discrimination task. J Neurophysiol. 1992 May; 67(5):1031-56.

[26] Recanzone GH, Merzenich MM, Jenkins WM. Frequency discrimination training engaging a restricted skin surface results in an emergence of a cutaneous response zone in cortical area 3a. J Neurophysiol. 1992 May; 67(5):1057-70.

[27] Wolpaw JR, Tennissen AM. Activity-dependent spinal cord plasticity in health and disease. Annu Rev Neurosci. 2001; 24:80743.

[28] Wolpaw JR. The complex structure of a simple memory. Trends Neurosci. 1997 Dec; 20(12):588-94.

[29] Bianconi R, van der MEULEN J. The response to vibration of the end organs of mammalian muscle spindles. J Neurophysiol. 1963 Jan; 26:177-90.

[30] Roll JP, Vedel JP, and Ribot E. Alteration of proprioceptive messages induced by tendon vibration in man: a microneurographic study. Exp Brain Res. 1989; 76(1):213-22.

[31] Age and gender effects on postural control measures. Arch Phys Med Rehabil. 1995 Oct 1; 76(10):961-5.

[32] Effect of Age and Activity on Knee Joint Proprioception 1 : American Journal of Physical Medicine \& Rehabilitation [Internet]. [Cited 2021 Jan 24]. Available from: https://journals.lww.com/ajpmr/Fulltext/1997/05000/Effect_of_Age_and_Activity_on_Knee_Joint.15.aspx?casa_token=01NS_U02CZIAAAAA:_aZOgJP4ZtM3Fo9JI1TuT6qVf2NfLOSNJAZiYiof7SMEypJuTeDpOuosop4EG1SdX831HzPb26hBmFZ5l-nAyD8Xgw

[33] Effects of combined sensory and muscular training on balance in Japanese older adults - ScienceDirect [Internet]. [Cited 2021 Jan 24]. Available from: https://www.sciencedirect.com/science/article/abs/pii/S0091743504002439

[34] Ramsbottom R, Ambler A, Potter J, Jordan B, Nevill A, Williams C. The Effect of 6 Months Training on Leg Power, Balance, and Functional Mobility of Independently Living Adults Over 70 Years Old. J Aging Phys Act. 2004 Oct 1; 12(4):497-510.

[35] Faber MJ, Bosscher RJ, Chin a Paw MJ, van Wieringen PC. Effects of Exercise Programs on fall and Mobility in Frail and PreFrail Older Adults: A Multicenter Randomized Controlled Trial. Arch Phys Med Rehabil. 2006 Jul 1; 87(7):885-96.

[36] Changes in power and force generation during coupled eccentric-concentric versus concentric muscle contraction with training and aging I SpringerLink [Internet]. [Cited 2021 Jan 24]. Available from: https://link.springer.com/article/10.1007/s00421-0080678-x

[37] High-volume, heavy-resistance strength training and muscle damage in young and older women I Journal of Applied Physiology [Internet]. [Cited 2021 Jan 24]. Available from: https://journals.physiology.org/doi/full/10.1152/jappl.2000.88.3.1112 